\title{
Immune response in a regressing compound dysplastic nevus
}

\author{
Ana Maria Abreu Velez', Charles J Douchy², Michael S. Howard ${ }^{1}$ \\ ${ }^{1}$ Georgia Dermatopathology Associates, Atlanta, Georgia, USA. ${ }^{2}$ North Atlanta Dermatology, Duluth, Georgia, USA \\ Corresponding author: Ana Maria Abreu Velez, E-mail: abreuvelez@yahoo.com
}

\begin{abstract}
Atypical (dysplastic) melanocytic nevi are considered for many as an intermediate step in a clinicopathologic spectrum. Regression is often seen. A 45 year old male presented with a hyperpigmented macule on the patient's back. Biopsies for hematoxylin and eosin (H\&E) examination and immunohistochemistry (IHC) analysis were performed. The H\&E staining demonstrated a compound dysplastic nevus. IHC stain shows stains with galectin 3, CD45, B-cell lymphoma 2 cyclooxygenase-2 ribosomal protein S6-ps240 (RIBO), CD68 and tyrosinase. Some stains were not only present in the tumor, but around it. We described a case of compound dysplastic nevi with a likely effectual early antitumoral response avoiding the growth to melanoma. The fundamental mechanism has not been elucidated, but our findings indicate a mix of inflammatory as well as immune response mediators maybe involved in the immune-based obliteration of melanocytes.
\end{abstract}

Keywords: Compound dysplastic nevus; Galectin 3; Ribosomal protein S6-ps240; BCL-2.

\section{INTRODUCTION}

Atypical (dysplastic) melanocytic nevi are considered for many as an intermediate step in a clinicopathologic spectrum from acquired melanocytic nevi to malignant melanoma.[1,2] Sometimes these nevi seem to be "self-regressing", but the manner in which the immune system produces these changes remains unknown. For many years, it has been thought that the immune response in melanocytic and other skin cancers was "not effective", and/or absent or anergic [1].

\section{CASE REPORT}

A 45 year old male presented for a skin examination. The dermatologist noted a hyperpigmented macule on the patient's back, and a skin biopsy for hematoxylin and eosin (H\&E) and immunohistochemistry (IHC) analysis was obtained.
Skin biopsies were taken for histology (H\&E) studies, for immunohistochemistry (IHC) and for direct immunofluorescence studies (DIF); our techniques were performed as previously described [2].

\section{Immunohistochemistry (IHC), Single and Double Color}

For our IHC testing, we utilized a dual endogenous peroxidase blockage, with the addition of a Dako Envision dual link (to assist in chromogen attachment). Our IHC staining was performed as previously described, using either single or double color techniques [2]. We then applied the chromogen 3,3-diaminobenzidine, and counterstained with hematoxylin. The samples were run in a Dako Autostainer Universal Staining System. Positive and negative controls were consistently performed. The staining was performed as previously described [2]. We performed IHC utilizing multiple monoclonal and polyclonal antibodies from Dako (Carpinteria, California,

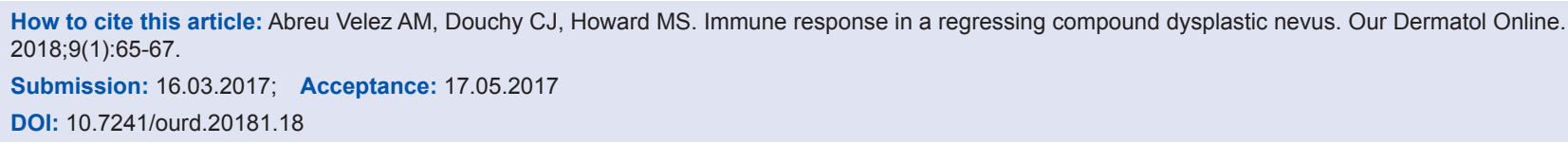


USA). We utilized the following mouse anti-human monoclonal antibodies: We utilized immune system cell markers, and other markers investigating cellular processes such as growth, proliferation, motility, and survival including galectin 3, CD45, B-cell lymphoma 2 (BCL-2), cyclooxygenase-2 (COX-2), ribosomal protein S6-ps240 (RIBO), CD68 and tyrosinase.

\section{RESULTS}

The H\&E staining demonstrated a melanocytic neoplasm, with melanocytes present as nests and single cells located along the dermal/epidermal junction (Fig. la, black arrow) (100X). Mild cytologic atypia was seen within these cells, but pagetoid spread of melanocytes was not appreciated. The melanocytes displayed bridging between adjacent rete ridges, and lamellar fibroplasia of collagen was present. The lesion appeared free of the specimen borders in the sections examined.

Tyrosinase was positive in the dysplastic nevi (Fig. 1b, brown staining; red arrow) (200X). Galectin 3 was also positive in melanocytic areas, mostly displaying cytoplasmic staining. COX-2 staining was positive on inflammatory cells around dermal vessels (Fig. lc, red staining; black arrow) (200X). Fig. ld shows colocalization of CD45 positive cells in red (red arrow) and COX-2 positive cells in brown (black arrow) (200X). RIBO and BCL-2 were both positive colocalizing in the dysplastic nevus and in the inflammatory cells under it. A few cells positive for CD68 were noted under the dysplastic nevus (image not shown), indicating the presence of antigen presenting cells in this area.

\section{DISCUSSION}

In this case we were able to see interactions between the melanocytic cells, the innate immune response, and the inflammatory response. Innate immunity is thought to provide a rapid, incomplete antimicrobial host defense until acquired immunity develops. COX-2 positive staining indicates an innate immunity response. The antigen presenting cells (CD68 staining) likely potentiate a specialized immune response, in combination with the CD45 positive cells.

Galectin-3 is a member of the lectin and the betagalactoside binding protein families. Galectin plays a role in cell-cell adhesion, cell-matrix interactions and macrophage activation, among other functions $[3,4]$. Other authors had studied the presence of galectin 3

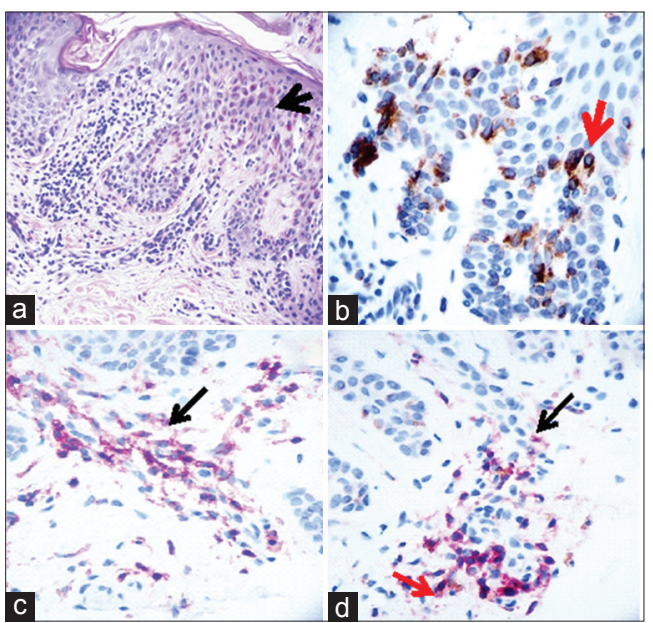

Figure 1: a. The H\&E staining demonstrated a melanocytic neoplasm, with melanocytes present as nests and single cells located along the dermal/epidermal junction (black arrow) (100X). Mild cytologic atypia was seen within these cells, but pagetoid spread of melanocytes was not appreciated. b. Positive IHC stain with tyrosinase was positive in the dysplastic nevi, brown staining; red arrow) (200X). c. Double color IHC stain shows a positive IHC stain with galectin 3 was positive in the melanocytes, mostly displaying cytoplasmic staining (brown stain). COX-2 staining was positive on inflammatory cells around dermal vessels (red staining; black arrow) (200X). d. shows colocalization of positive double color with IHC CD45 in red (red arrow) and COX-2 positive cells in brown (black arrow) (200X).

in differential melanocytic lesions, and concluded that strong galectin 3 expression was associated with an improved overall survival from melanocytic lesions. Other studies had shown contradictory results, showing that melanocytes accumulate galectin-3 with tumor progression, particularly in the nuclei $[3,4]$. Here Galectin 3 is likely allowing a flux of the immune cells into the nevus. On the other hand, BCL-2 regulates cell death (apoptosis), by either inducing (pro-apoptotic) or inhibiting (antiapoptotic) functions [5]. BCL-2 expression in dysplastic nevi likely signals an immune response against the nevus by apoptosis, and/or pro-apotosis [5].

In regards to the positivity of COX-2, it represents an inducible enzyme involved in the production of prostaglandins and thromboxanes during inflammation. There is evidence of COX-2 acting to induce flux of immune cells into melanocytic lesions. However, other lines of evidence indicate that increased expression of COX-2 plays a functional role in the development and progression of malignant melanocytic cells [6]. We suggest that COX-2 and BCL-2 roles could change on a case-by case basis, either promoting immunity and/ or inhibiting it in dysplastic nevi. Given the fact that most pre-melanoma and melanoma lesions are quickly excised, verification of these precise functions would require creating melanoma cell line animal models, 
then testing the markers throughout melanoma progression.

In conclusion, here we present some inflammatory and immune response in a regressing dysplastic nevus that maybe an example of an efficient early antitumoral response preventing the development of neoplasia. The immunosurveillance of this compound dysplastic nevi seem to play of major importance given the possibility to progress to melanoma. Specifically, the area around the tumor and the immune system cells includes an innate and a specialized immune response as well as inflamation.

\section{Abbreviations}

Hematoxylin and eosin (H\&E), immunohistochemistry (IHC), ribosomal protein S6-ps240 (RIBO), B-cell lymphoma 2 gene (BCL-2), cyclooxygenase-2 (COX-2).

\section{ACKNOWLEDGEMENT}

Jonathan S. Jones, HT(ASCP) at Georgia Dermatopathology Associates provided excellent technical assistance.

\section{REFERENCES}

1. Clark WH Jr, Elder DE, Guerry D $4^{\text {th }}$, Epstein MN, Greene MH, Van Horn M. A study of tumor progression: The precursor lesions of superficial spreading and nodular melanoma. Hum Pathol. 1984;15:1147-65.

2. Abreu Velez AM, Googe PB Jr, Kim J, Howard MS. Basal cell carcinoma with sebaceous differentiation markers. North Am J Med; 2015.7:275-80.

3. Brown ER, Doig T, Anderson N, Brenn T, Doherty V, Xu Y, et al. Association of galectin-3 expression with melanoma progression and prognosis. Eur J Cancer. 2012;48:865-74.

4. Prieto VG, Mourad-Zeidan AA, Melnikova V, Johnson MM, Lopez A, Diwan AH, et al. Galectin-3 expression is associated with tumor progression and pattern of sun exposure in melanoma. Clin Cancer Res. 2006;12:6709-15.

5. Becker JC, thor Straten P, Andersen MH. The Paradoxical association between $\mathrm{Bcl}-2$ expression and prognosis: Does the immune system make the difference? Cancer Res. 2005;65:7.

6. Goulet AC, Einsphar JG, Alberts DS, Beas A, Burk C, Bhattacharyya A, et al. Analysis of cyclooxygenase 2 (COX-2) expression during malignant melanoma progression. Cancer Biol Ther. 2003;2:713-8.

Copyright by Abreu Velez, et al. This is an open-access article distributed under the terms of the Creative Commons Attribution License, which permits unrestricted use, distribution, and reproduction in any medium, provided the original author and source are credited.

Source of Support: Georgia Dermatopathology Associates, Atlanta,

Georgia, USA, Conflict of Interest: None declared. 